\title{
Produção e qualidade de couve-flor cultivar Teresópolis Gigante em função de doses de nitrogênio
}

\author{
Cristiaini Kano; ${ }^{1}$ Ariane C Salata ${ }^{2}$; Andréa RO Higuti²; Amanda Regina Godoy³; Antonio Ismael Inácio \\ Cardoso $^{2}$; Regina Marta Evangelista ${ }^{4}$ \\ ${ }^{1}$ APTA-Pólo Leste Paulista, C. Postal 01, 13910-000 Monte Alegre do Sul-SP; ${ }^{2}$ UNESP-FCA, Dep ${ }^{\text {to }}$ Prod. Vegetal, Setor Horticultura, C. \\ Postal 237, 18610-307 Botucatu-SP; ${ }^{3}$ UFMS, C. Postal 112, 79560-000 Chapadão do Sul-MS; ${ }^{4}$ UNESP-FCA, Dep ${ }^{\text {to }}$ Gestão e Tecnologia \\ Agroindustrial; criskano@hotmail.com; acsalata@fca.unesp.br; anreiko_higuti@hotmail.com; amanda@ufms.br; ismaeldh@fca.unesp. \\ br; evangelista@fca.unesp.br
}

\section{RESUMO}

O objetivo deste trabalho foi avaliar a influência de doses de nitrogênio em cobertura na produção e na qualidade da couve-flor cultivar Teresópolis Gigante. O experimento foi desenvolvido na Fazenda Experimental São Manuel, pertencente à UNESP-FCA, de abril a agosto de 2006. O delineamento experimental utilizado foi blocos casualizados com cinco tratamentos $(0 ; 75 ; 150 ; 225$ e $300 \mathrm{~kg}$ $\mathrm{ha}^{-1} \mathrm{de} \mathrm{N}$ ) e quatro repetições com dez plantas por parcela. As características avaliadas foram a massa e o diâmetro médio da "cabeça", número de folhas por planta, teor de nitrogênio na "cabeça", $\mathrm{pH}$, acidez titulável, sólidos solúveis, ácido ascórbico e açúcares redutores. Observou-se aumento linear na massa média das "cabeças" e no teor de nitrogênio nas "cabeças" com o aumento da dose de nitrogênio, no entanto, sem afetar o diâmetro das mesmas e o número de folhas por planta. Quanto às características de qualidade observou-se um aumento linear no $\mathrm{pH}$ com o aumento da dose de nitrogênio, porém, para as demais características não houve diferença estatística entre as doses de nitrogênio avaliadas.

Palavras-chave: Brassica oleracea var. botrytis, adubação nitrogenada, qualidade pós-colheita.

\begin{abstract}
Cauliflower cultivar Teresópolis Gigante production and quality depending on nitrogen levels

The purpose of the present research was to evaluate the influence of nitrogen levels in covering on yield and quality of cauliflower, cultivar Teresópolis Gigante. The experiment was carried out at Universidade Estadual Paulista, in São Paulo State, Brazil. The evaluated characteristics were curd weight, curd diameter, number of leaves per plant, curd nitrogen content, texture, $\mathrm{pH}$, acidity, total soluble solids, ascorbic acid and reducing sugars. A linear increase in curd weight and nitrogen content in curd was observed with increasing nitrogen levels, but without affecting curd diameter and number of leaves per plant. For the quality characteristics we observed linear increase in $\mathrm{pH}$ with increasing nitrogen levels but to the other characteristics there were no statistical differences among the nitrogen levels evaluated.
\end{abstract}

Keywords: Brassica oleracea var. botrytis, nitrogen fertilization, postharvest quality.

(Recebido para publicação em 9 de julho de 2009; aceito em 19 de outubro de 2010) (Received on July 9, 2009; accepted on October 19, 2010)

\begin{abstract}
A couve-flor (Brassica oleracea var. botrytis), pertencente à família das Brassicáceas, pode ser produzida durante o ano inteiro, em praticamente todo o Brasil, graças aos programas de melhoramento genético, que produziram cultivares e híbridos adaptados à alta temperatura. É uma hortaliça com características nutracêuticas e a parte comestível é composta por uma inflorescência imatura que pode ter coloração branca, creme, amarela, e mais recentemente roxa e verde (May et al., 2007; Filgueira, 2008).

A qualidade e a produtividade da couve-flor são influenciadas pelo manejo da adubação e pelas condições climáticas, dentre outros fatores. Dos nutrientes essenciais fornecidos por meio da adubação química, destacamse o nitrogênio, fósforo e potássio, que
\end{abstract}

devem ser aplicados em níveis compatíveis às exigências de cada cultura e ao método de adubação utilizado.

O nitrogênio na planta possui função estrutural, participa dos processos de absorção iônica, fotossíntese, respiração, multiplicação e diferenciação celular. Estimula a formação e o desenvolvimento de gemas floríferas e frutíferas. Em geral, sua deficiência causa o amarelecimento inicialmente das folhas mais velhas das plantas entre outros sintomas e o seu excesso causa o atraso no florescimento (Malavolta, 1980; Marschner, 1995), aspecto importante a ser considerado para a cultura da couveflor, que tem como parte comercial a "inflorescência".

Os macronutrientes potássio e nitrogênio são extraídos em maiores quantidades pela couve-flor, mas, expe- rimentalmente, nitrogênio e fósforo são os nutrientes que têm fornecido maiores respostas em produtividade. Após o transplante, o fornecimento parcelado de nitrogênio promove crescimento vegetativo vigoroso, favorecendo a produtividade, existindo correlação direta entre a superfície foliar e o desenvolvimento da "cabeça", de forma que plantas maiores originam "cabeças" de maior tamanho (Filgueira, 2008). Conforme Marschner (1995), o nitrogênio é o nutriente que pode alterar a composição da planta mais do que qualquer outro nutriente mineral.

Segundo Furlani et al. (1978) e Sánchez et al. (2001), o nitrogênio é o primeiro nutriente em \% da matéria seca tanto na folha como na "cabeça" da couve-flor. Também Takeishi \& Cecílio Filho (2007) verificaram que o nitro- 
gênio foi o nutriente mais acumulado na couve-flor 'Verona', com a seguinte ordem decrescente: $\mathrm{N}>\mathrm{K}>\mathrm{Ca}>\mathrm{S}>\mathrm{P}>\mathrm{Mg}$. Ainda com este híbrido Verona, Pôrto (2009) obteve maior acúmulo de $\mathrm{N}$, seguido do K e do P. Porém, Homa et al. (1969) e Oliveira et al. (1971) verificaram que em couve-flor a absorção de macronutrientes obedeceu a mesma sequência de acúmulo de matéria seca, sendo os nutrientes absorvidos pelas plantas na seguinte seqüência: $\mathrm{K}>\mathrm{N}>\mathrm{Ca}>\mathrm{S}>\mathrm{Mg}>\mathrm{P}$ mostrando que $\mathrm{o}$ nitrogênio foi o segundo nutriente mais extraído pela couve-flor, reforçando a importância desse nutriente para essa cultura.

Para solos de fertilidade mediana ou baixa, sugere-se aplicação de 80 ; $350-500$ e $150-200 \mathrm{~kg} \mathrm{ha}^{-1}$ de N, $\mathrm{P}_{2} \mathrm{O}_{5}$ e $\mathrm{K}_{2} \mathrm{O}$, respectivamente aplicados no sulco de transplante das mudas (Filgueira, 2008). Raij et al. (1996) recomendam 60; 400-600; 180-240 de N, $\mathrm{P}_{2} \mathrm{O}_{5}$ e $\mathrm{K}_{2} \mathrm{O}$, respectivamente e adubação de cobertura com 15 a $200 \mathrm{~kg} \mathrm{ha}^{-1}$ de $\mathrm{N}$ e 60-120 $\mathrm{kg} \mathrm{ha}^{-1}$ de $\mathrm{K}_{2} \mathrm{O}$, parcelando em quatro vezes, aos $15,30,45$ e 60 dias após o transplante.

Batal et al. (1997), ao estudarem doses de nitrogênio no cultivo de couve-flor, verificaram que a máxima massa média de "cabeça" e de produção foram obtidas nas maiores doses de nitrogênio (269 e $381 \mathrm{~kg} \mathrm{ha}^{-1}$ de N) aplicadas em solo arenoso e argiloso, respectivamente.

As espécies da família Brassicaceae apresentam grande capacidade de resposta à adubação nitrogenada, verificando-se aumentos na produtividade das culturas com até $300 \mathrm{~kg} \mathrm{ha}^{-1} \mathrm{de} \mathrm{N}$ (Trani et al., 2007). Camargo et al. (2008), ao avaliarem doses de 100 a $250 \mathrm{~kg} \mathrm{ha}^{-1} \mathrm{de}$ $\mathrm{N}$ em couve-flor de inverno, verificaram que as doses de nitrogênio aumentaram de modo linear à produtividade total e à massa média da "cabeça", sem influenciar o diâmetro da "cabeça".

A falta de informações relacionadas à adubação da couve-flor evidencia a necessidade do desenvolvimento de pesquisas relacionadas à determinação de doses de nutrientes que proporcionem aumento na produtividade e melhoria na qualidade físico química das "cabeças".

O objetivo deste trabalho foi ava- liar o efeito de doses de nitrogênio em cobertura na produção e qualidade da couve-flor.

\section{MATERIAL E MÉTODOS}

O experimento foi conduzido na Fazenda Experimental São Manuel, no município de São Manuel-SP, pertencente à UNESP em Botucatu.

Foi utilizado no experimento um Latossolo Vermelho Distrófico Típico (Embrapa, 1999) com as seguintes características químicas: $\mathrm{pH}\left(\mathrm{CaCl}_{2}\right)=6,0$; $\mathrm{P}_{\text {resina }}=69 \mathrm{mg} \mathrm{dm}^{-3}$; matéria orgânica $=9$ $\mathrm{g} \mathrm{dm}^{-3} ; \mathrm{V} \%=75 ; \mathrm{H}+\mathrm{Al}=13 \mathrm{mmol}_{\mathrm{c}} \mathrm{dm}^{-3}$; $\mathrm{K}=0,9 \mathrm{mmol}_{\mathrm{c}} \mathrm{dm}^{-3} ; \mathrm{Ca}=28 \mathrm{mmol}_{\mathrm{c}}^{\mathrm{c}} \mathrm{dm}^{-3}$; $\mathrm{Mg}=9 \mathrm{mmol}_{\mathrm{c}}^{\mathrm{c}} \mathrm{dm}^{-3} ; \mathrm{SB}=38 \mathrm{mmol}_{\mathrm{c}} \mathrm{dm}^{-3}$ e CTC $=51 \mathrm{mmol}_{c} \mathrm{dm}^{-3}$.

A adubação de plantio foi realizada no dia 10/05/06, aplicando-se o equivalente a $50 \mathrm{t} \mathrm{ha}^{-1}$ de composto orgânico, $60 \mathrm{~kg} \mathrm{ha}^{-1}$ de N, $200 \mathrm{~kg} \mathrm{ha}^{-1}$ de $\mathrm{P}_{2} \mathrm{O}_{5}$ e $200 \mathrm{~kg} \mathrm{ha}^{-1} \mathrm{de} \mathrm{K}_{2} \mathrm{O}$ utilizando como fonte desses nutrientes o sulfato de amônio e o formulado 4-20-20.

O delineamento experimental utilizado foi blocos ao acaso, com cinco tratamentos e quatro repetições com dez plantas por parcela. Os tratamentos foram constituídos pelas doses de $0 ; 75$; $150 ; 225$ e $300 \mathrm{~kg} \mathrm{ha}^{-1}$ de N em cobertura, sendo a dose de $150 \mathrm{~kg} \mathrm{ha}^{-1}$ de N a recomendada por Raij et al. (1996) para essa cultura.

As doses de nitrogênio foram aplicadas semanalmente em cobertura, parceladas em nove vezes, de 29/05 a 27/07/06, utilizando-se como fonte desse nutriente a uréia. Foram também realizadas três aplicações foliares de ácido bórico e molibdato de amônio, seguindo a recomendação de Raij et al. (1996) para essa cultura.

A semeadura foi realizada em 10/04/06 em bandejas de poliestireno expandido de 128 células contendo substrato comercial utilizando a cultivar de inverno Teresópolis Gigante, que, conforme citação de May et al. (2007), possui plantas vigorosas, bem desenvolvidas, de inflorescência grande, compacta e de coloração branca e ciclo de 140-150 dias da semeadura.

As mudas foram transplantadas em canteiros em 12/05/06, quando estavam emitindo a segunda folha definitiva no espaçamento de $0,8 \times 0,6 \mathrm{~m}$. O controle fitossanitário foi realizado com inseticida piretróide para o controle de pulgão e a irrigação foi realizada por aspersão em média a cada dois dias com cerca de $8 \mathrm{~mm}$.

As colheitas, num total de cinco, abrangeram de 04 a 24/08/06, sendo que em cada colheita foram avaliados o número de folhas por planta, a massa média da "cabeça" (sem folhas e com o talo cortado rente à "cabeça") e o diâmetro médio da "cabeça".

Após as avaliações de produção das "cabeças", essas foram levadas para o laboratório de fisiologia pós-colheita de frutas e hortaliças da UNESP em Botucatu-SP onde foram lavadas com água corrente e posteriormente com água destilada para a realização das seguintes análises: a) pH e acidez titulável (\%), conforme técnicas desenvolvidas pelo Instituo Adolfo Lutz, publicadas em Brasil (2005); b) sólidos solúveis, realizado conforme recomendação feita pela AOAC (1992) e os resultados expressos em ${ }^{\circ} \mathrm{Brix}$; c) açúcares redutores totais, determinados pelo método descrito por Somogyi e adaptado por Nelson (1944), sendo os resultados expressos em \%, d) ácido ascórbico determinado pelo método de Tillmans padronizado pelo Instituo Adolfo Lutz, publicado em Brasil (2005) e expresso em mg 100 $\mathrm{mL}^{-1} \mathrm{e}$ teor de nitrogênio $\left(\mathrm{g} \mathrm{kg}^{-1}\right)$ conforme Malavolta et al. (1997) em que para essa análise, as "cabeças" de couve-flor foram cortadas e colocadas para secar a $65^{\circ} \mathrm{C}$ em estufa de circulação forçada de ar até atingirem massa constante. Posteriormente, cada amostra passou pela moagem no moinho tipo Willey e pela digestão sulfúrica para a obtenção do extrato visando a determinação de nitrogênio.

As avaliações de qualidade foram realizadas com as "cabeças" colhidas no dia 21/08/06. Porém, nesta data, não houve número suficiente de "cabeças" na dose de $300 \mathrm{~kg} \mathrm{ha}^{-1}$ de N, (T5), por isso, não foi possível avaliar as características de qualidade, sendo consideradas para essas avaliações apenas as "cabeças" obtidas do T1 ao T4.

Os dados foram submetidos à análise de variância pelo teste $\mathrm{F}$ e quando houve efeito significativo para tratamentos, realizou-se a análise de regressão em 
função das doses de nitrogênio utilizando-se o programa estatístico SISVAR (Ferreira, 1999).

\section{RESULTADOS E DISCUSSÃO}

A primeira colheita foi realizada com 116 dias após a semeadura e a última aos 136 dias, sem diferença entre os tratamentos. Portanto, apesar das elevadas doses de nitrogênio utilizadas, foram obtidos menores ciclos em relação ao citado por May et al. (2007) para a cultivar Teresópolis Gigante: 140 a 150 dias após a semeadura.

Os tratamentos avaliados não influenciaram o diâmetro médio da "cabeça" bem como o número de folhas por planta. O diâmetro médio da "cabeça" variou de 19,2 (T1= sem nitrogênio em cobertura) a $21,8 \mathrm{~cm}\left(\mathrm{~T} 5=300 \mathrm{~kg} \mathrm{ha}{ }^{-1}\right.$ de $\mathrm{N}$ ), com uma média de $20,5 \mathrm{~cm}(\mathrm{CV}=$ $6,5 \%$ ), valores semelhantes aos obtidos por Camargo et al. (2008) e por Pizetta et al. (2005) que relataram média de diâmetro de 21,06 e 18,2 cm, respectivamente. Camargo et al. (2008) e Mello et al. (2009) também não verificaram a influência de doses crescentes de nitrogênio no diâmetro médio da "cabeça" de couve-flor híbridos 'Julia' e 'Sharon', respectivamente.

O número de folhas por planta variou de 23 (T5) a 24 (T1) com uma média de 23,4 folhas por planta $(\mathrm{CV}=$ $5,7 \%$ ), valor semelhante ao encontrado por Almeida et al. (2007) para a cultivar Teresópolis Gigante que foi de 23 folhas por planta.

Já a massa média da "cabeça" ajustou-se estatisticamente ao modelo linear em função dos tratamentos (Figura 1), demonstrando o aumento da massa com o aumento da quantidade de nitrogênio fornecida, concordando com os resultados obtidos por Camargo et al. (2008) que também obtiveram aumento linear com doses de nitrogênio variando de 100 a $250 \mathrm{~kg} \mathrm{ha}^{-1}$ de $\mathrm{N}$ e por Batal et al. (1997) ao avaliarem doses de $101 \mathrm{a}$ $381 \mathrm{~kg} \mathrm{ha}^{-1}$ de $\mathrm{N}$, indicando resposta da couve-flor à adubação nitrogenada. De acordo com a Figura 1, estima-se que para cada $100 \mathrm{~kg} \mathrm{ha}^{-1}$ de N obtém-se um aumento de 177 gramas por "cabeça" e observa-se que a partir da dose de 150 $\mathrm{kg} \mathrm{ha}^{-1}$ de N, a massa média da "cabeça"

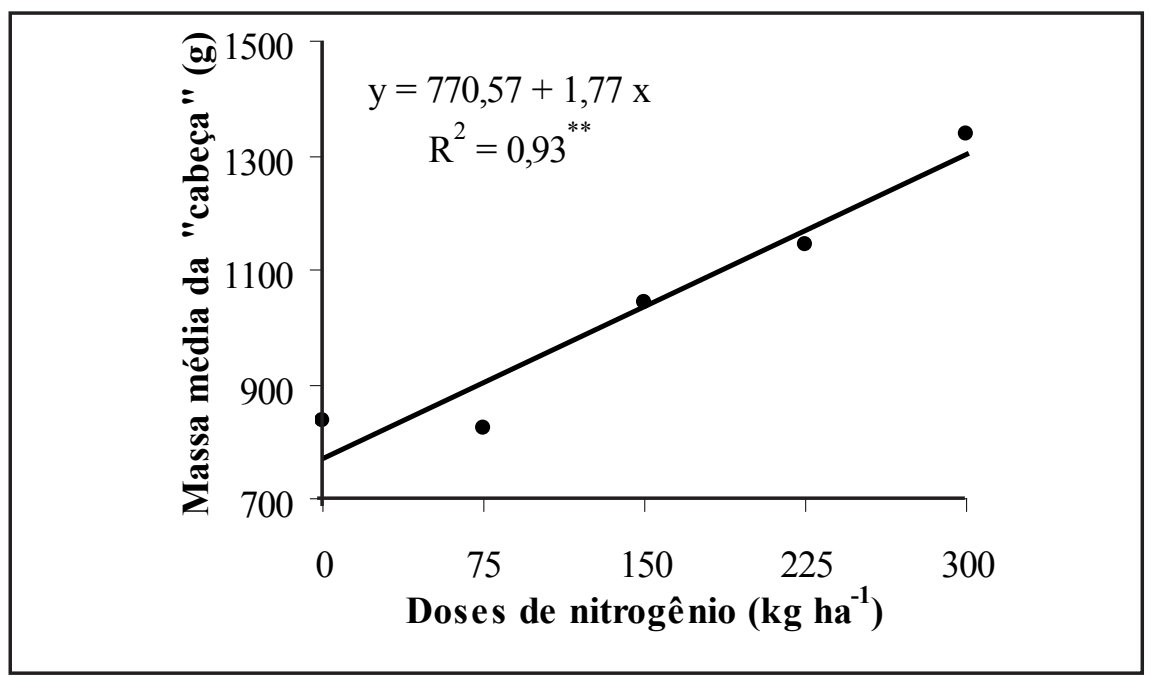

Figura 1. Massa média da "cabeça" de couve-flor $(\mathrm{g})$ em função das doses de nitrogênio (average weight $(\mathrm{g})$ of cauliflower curd in function of nitrogen levels). Botucatu, UNESP, 2006.

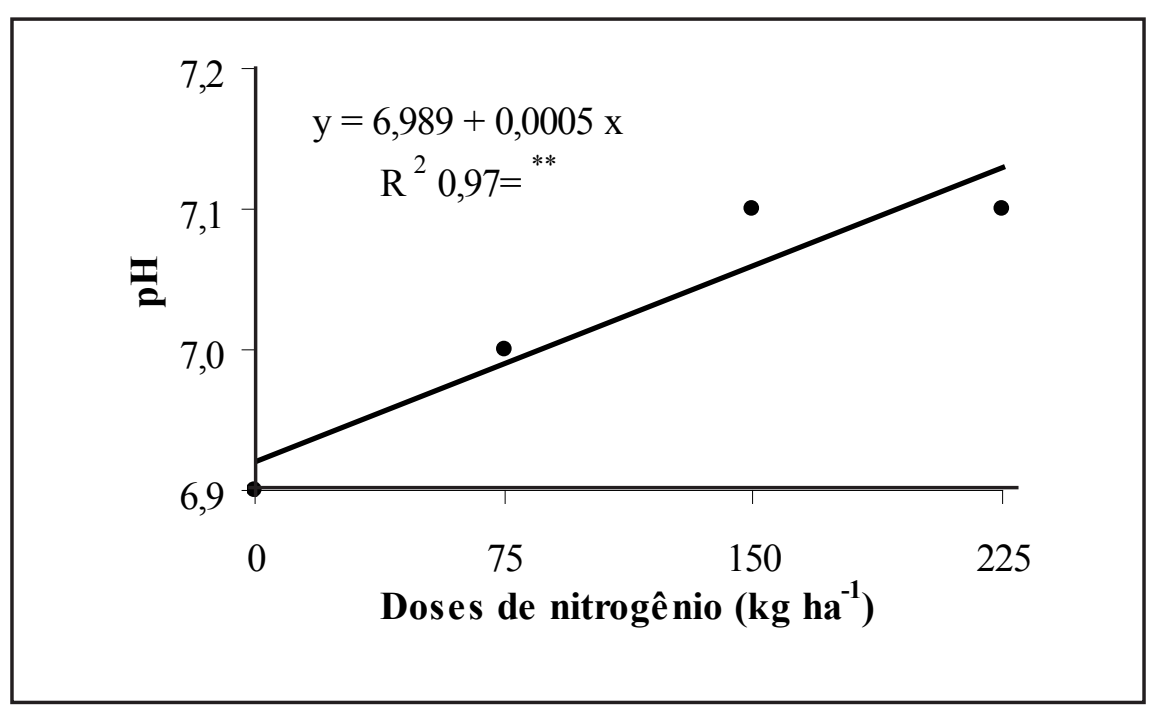

Figura 2. Média dos valores de pH em "cabeças" de couve-flor em função das doses de nitrogênio (average $\mathrm{pH}$ values in cauliflower curd in function of nitrogen levels). Botucatu, UNESP, 2006.

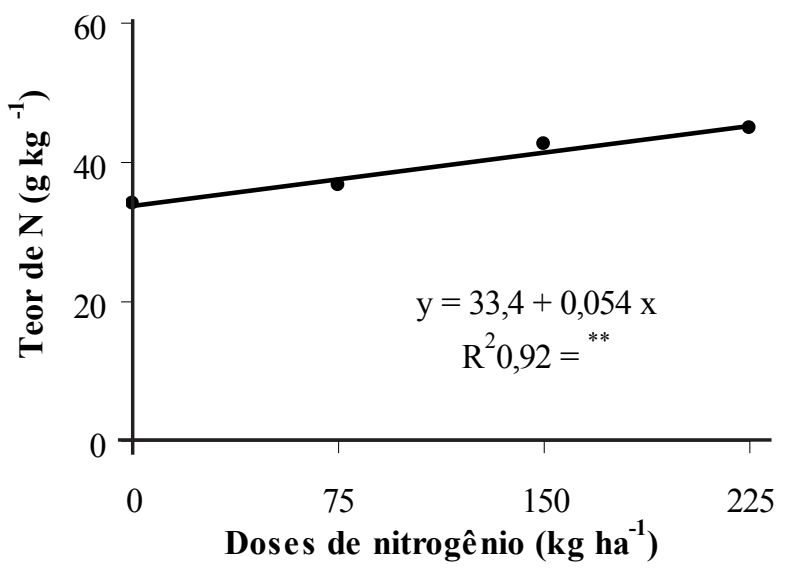

Figura 3. Teor de nitrogênio nas "cabeças" de couve-flor em função das doses de nitrogênio (nitrogen content in cauliflower curd in function of nitrogen levels). Botucatu, UNESP, 2006. 
obtida foi maior que $1 \mathrm{~kg}$.

Mello et al. (2009) não observaram diferença na massa média de "cabeça" com doses de nitrogênio em cobertura variando de 100 a $250 \mathrm{~kg} \mathrm{ha}^{-1}$. Porém, estes autores realizaram o trabalho no verão e com um material mais precoce. A cultivar utilizada neste experimento, Teresópolis Gigante, é uma das mais tardias existentes no mercado e, talvez, necessite de maiores quantidades de nitrogênio e maior parcelamento. $\mathrm{O}$ recomendado é a divisão em três vezes, sendo que nesta pesquisa as doses avaliadas foram parceladas em nove vezes em busca de melhor aproveitamento de nitrogênio pelas plantas.

Considerando-se que a dose recomendada por Raij et al. (1996) é de $150 \mathrm{~kg} \mathrm{ha}^{-1}$ de $\mathrm{N}$, percebeu-se que a cultura pode ter seu potencial produtivo aumentado com a aplicação de doses de nitrogênio superiores ao recomendado para o estado de São Paulo. Esta dose recomendada pode não ser a ideal para todas as condições de cultivo. Sendo a cultura originalmente adaptada ao cultivo de outono-inverno, para estas condições ideais pode haver a necessidade de maiores quantidades de nutrientes para se obter o máximo desenvolvimento das "cabeças".

Não foram obtidas diferenças significativas para as características de qualidade avaliadas, exceto para o $\mathrm{pH}$. Observou-se aumento linear no valor do $\mathrm{pH}$ com aumento nas doses de nitrogênio (Figura 2). Porém, apesar de significativo, este aumento foi muito pequeno, passando de 6,9 (dose 0 ) para 7,1 nas maiores doses analisadas (150 e $225 \mathrm{~kg} \mathrm{ha}^{-1}$ de N).

$\mathrm{O}$ açúcar redutor variou de 3,9 a 4,3, com média de 4,0\% (CV=7,3\%). Nilsson (1980) também não observou influencia da aplicação de nitrogênio (150 e $300 \mathrm{~kg} \mathrm{ha}^{-1}$ de N) nos teores de açúcares de couve-flor. Já Kunwar \& Pandey (1994) ao avaliarem quatro níveis de $\mathrm{N}\left(0,100,150\right.$ e $\left.200 \mathrm{~kg} \mathrm{ha}^{-1}\right)$, observaram que a máxima quantidade de açúcares totais $(4,2 \%)$ foi obtida na dose de $100 \mathrm{~kg} \mathrm{ha}^{-1}$.

Os teores de ácido ascórbico variaram de 47,9 a 49,5, com média de 49,1 $\mathrm{mg} 100 \mathrm{~mL}^{-1}(\mathrm{CV}=11,4 \%)$. Em revisão feita por Mozafar (1993) sobre os efeitos da adubação nitrogenada no conteúdo de vitaminas em plantas, indicou que a resposta da adubação nitrogenada em hortaliças é variada, resultando em efeito positivo, negativo e sem efeito na concentração de várias vitaminas dependendo da cultura. Dude et al. (1975) e Kappel (1977) citados por Mozafar (1993), assim como também, Maurya et al. (1992) observaram aumento na concentração de ácido ascórbico quando estes aumentaram a aplicação de nitrogênio na cultura da couve-flor. Randhawa \& Bhail (1976) verificaram aumento nos teores de ácido ascórbico quando os níveis de N, P e B eram de 120, 40 e $20 \mathrm{~kg} \mathrm{ha}^{-1}$, respectivamente. Ainda em couve-flor, Fritz et al. (1979) citado por Mozafar (1993), não encontraram efeito na concentração de ácido ascórbico, no entanto, Jha et al. (1973) e Lisiewska \& Kmiecik (1996) observaram diminuição nos teores com aumento da fertilização de 60 para $120 \mathrm{~kg} \mathrm{ha}^{-1}$ de N. Essa divergência entre autores pode ser devido à quantidade de fertilizantes utilizados por cada um ou até mesmo pela interação entre as condições climáticas e a adubação utilizada. Segundo Podsedek (2007), o conteúdo de ácido ascórbico em Brassica varia significativamente entre e dentro de subespécies. Em sua revisão foram observados valores que variaram de 17,2 a 81,0 mg $100 \mathrm{~g}^{-1}$, sendo a diferença do menor para o maior valor de quatro vezes. O teor médio observado neste trabalho (49,1 mg 100 $\mathrm{mL}^{-1}$ ) está entre os valores citados por este autor.

Os teores de sólidos solúveis variaram de 6,7 a 7,2, com média de $6,9{ }^{\circ}$ Brix $(\mathrm{CV}=7,7 \%)$, valor próximo ao obtido por Brackmann et al. (2005) que foi de 6,6 ${ }^{\circ}$ Brix, também em "cabeças" de couve-flor Teresópolis Gigante.

A acidez titulável variou de 0,13 a 0,15 , com média de $0,14 \%$, porém estes teores não foram influenciados pela concentração de nitrogênio utilizada. Os teores de acidez observados são superiores aos citados por Jha et al. (1973) com valores em torno de $0,092 \%$, sendo que estes autores observaram diminuição na acidez com aumento da fertilização com nitrogênio.

Em relação ao teor de nitrogênio encontrado nas "cabeças", a quantidade variou de 33,8 a 44,8 $\mathrm{g} \mathrm{kg}^{-1}$, com média de $39,47 \mathrm{~g} \mathrm{~kg}^{-1}$ ajustando-se estatisticamente ao modelo linear (Figura 3), com valores semelhantes ao encontrado por Furlani et al. (1978) e por Mello et al.(2009) que foi de 38,9 e 37,0 $\mathrm{g} \mathrm{kg}^{-1}$ de $\mathrm{N}$, respectivamente. Camargo et al. (2008) também verificaram aumento linear no teor de nitrogênio com o aumento das doses de $\mathrm{N}$, porém com valores pouco inferiores $\left(29,4 \mathrm{~g} \mathrm{~kg}^{-1}\right.$ para $100 \mathrm{~kg} \mathrm{ha}^{-1}$ de $\mathrm{N}$ até $39,8 \mathrm{~g} \mathrm{~kg}^{-1}$ para $250 \mathrm{~kg} \mathrm{ha}^{-1} \mathrm{de} \mathrm{N}$ ).

Nas condições em que o experimento foi conduzido, observou-se que a adubação nitrogenada favoreceu a obtenção de "cabeças" mais pesadas, no entanto, sem afetar o diâmetro das mesmas, o número de folhas por planta bem como a maioria das características físico-químicas da couve-flor, com exceção do pH onde se obteve pequeno aumento linear com o aumento das doses de nitrogênio.

\section{AGRADECIMENTOS}

À Capes e à FAPESP pela concessão de bolsas de pós-graduação.

\section{REFERÊNCIAS}

ALMEIDA K; CARVALHO GJ; GOMES LAA; DUARTE WF; FONTANETTI A. 2007. Produção orgânica de couve-flor em sistema de plantio direto e convencional. Revista Brasileira de Agroecologia 2: 1216-1219.

ASSOCIATION OF OFFICIAL ANALYTICAL CHEMISTRY. 1992. Official methods of analysis of the association of official analytical chemistry. Washington: AOAC. 1015p.

BATAL KM; GRANBERRY DM; MULLINIX JUNIOR BG. 1997. Nitrogen, magnesium, and boron applications affect cauliflower yield, curd mass, and hollow stem disorder. Hortscience 32: 75-78.

BRACKMANN A; TREVISAN JN; MARTINS GAK; FREITAS ST; MELLO AM. 2005. Qualidade pós-colheita de couve-flor 'Teresópolis Gigante' tratada com etileno, absorvedor de etileno e 1-metilciclopropeno. Ciência Rural 35: 1444-1447.

BRASIL. 2005. Ministério da Saúde. Agência Nacional de Vigilância Sanitária. Métodos físico-químicos para análise de alimentos. Brasília, Ministério da Saúde. 1018p.

CAMARGO MS; MELLO SC; FOLTRÁN DE; CARMELLO QAC. 2008. Produtividade e podridão parda em couve-flor de inverno influenciadas pelo nitrogênio e boro. Bragantia 67: 371-375.

EMPRESA BRASILEIRA DE PESQUISA 
AGROPECUÁRIA. 1999. Sistema brasileiro de classificação de solos. Brasília: Embrapa. 412 p.

FERREIRA DF. 1999. Sistema de análise de variância (SISVAR) versão 4.6. Lavras: Universidade Federal de Lavras. CD-ROM.

FILGUEIRA FAR. 2008. Novo manual de olericultura: agrotecnologia moderna na produção e comercialização de hortaliças. Viçosa: UFV. 421p.

FURLANI, AMC; FURLANI, PR; BATAGLIA, OC; HIROCE, R; GALLO, JR. 1978. Composição mineral de diversas hortaliças. Bragantia 37: 33-34.

HOMA, PM; HAAG, HP; SARRUGE, JR. 1969. Nutrição mineral de hortaliças. II. Absorção de nutrientes pela cultura de couve-flor. $O$ solo 1: 9-16.

JHA J; DWIVEDI BK; PRASAD B. 1973. Effect of nitrogen fertilizer with and without organic manure on the quality of cauliflower. Journal of the Institution of Chemists 45: 26-28.

KUNWAR R; PANDEY CB. 1994. Growth, yield quality and macronutrient status of cauliflower (Brassica oleracea var. botrytis) in relation to nitrogen and potassium fertilization. Haryana Journal of Horticultural Science 23: 229-234.

MALAVOLTA E. 1980. Elementos de nutrição mineral de plantas. São Paulo: Agronômica Ceres. 251p.

MALAVOLTA E; VITTI GC; OLIVEIRA SA. 1997. Avaliação do estado nutricional das plantas, princípios e aplicações. Piracicaba: Potafós. 319p.

MARSCHNER H. 1995. Mineral nutrition of higher plants. London: Academic Press. 889p.

MAURYA AN; CHAURASIA SNS; REDDY YRM. 1992. Effect of nitrogen and molybdenum levels on growth, yield and quality of cauliflower (Brassica oleracea var. botrytis) cv. Snowball-16. Haryana Journal of Horticultural Science 21: 232-235.

MAY A; TIVELLI SW; VARGAS PF; SAMRA AG; SACCONI LV; PINHEIRO MQ. 2007. A cultura da couve-flor. Boletim Técnico IAC 200: 1-36.

MELLO SC; CAMARGO MS; VIVIAN R; NASCIMENTO TS; OLIVEIRA ES; BERTANHA R. 2009. Nitrogênio e boro na produção e incidência de haste oca em couveflor 'Sharon'. Bragantia 68: 761-764.

MOZAFAR A. 1993. Nitrogen fertilizers and the amount of vitamins in plants: A review. Journal of Plant Nutrition 16: 2479-2506.

NELSON N. 1944. A photometria adaptation of Somogyi method for determination of glicose. Journal Biological Chemistry 31: 159-161.

NILSSON T. 1980. The influence of soil type, nitrogen and irrigation on yield, quality and chemical composition of cauliflower. Swedish Journal Agricultural Research 10: 65-75.

OLIVEIRA GD; FERNANDES PD; SARRUGE JR; HAAG HP. 1971. Nutrição mineral de plantas. XIII. Extração e absorção de macronutrientes pelas hortaliças. $O$ solo 1 : 7-12.

PIZETTA LC; FERREIRA ME; CRUZ MCP; BARBOSA JC. 2005. Resposta de brócolis, couve-flor e repolho à adubação com boro em solo arenoso. Horticultura Brasileira 23: 51-56.

PODSEDEK A. 2007. Natural antioxidants and antioxidant capacity of Brassica vegetables: a review. Food Science and Technology 40: $1-11$.

PÔRTO DRQ. 2009. Densidades populacionais e épocas de plantio na cultura da couve-flor, hibrido Verona 284. Jaboticabal: UNESPFCAV. 74p. (Tese doutorado).

RAIJ BVan; CANTARELLA H; QUAGGIO JA; FURLANI AMC. 1996. Recomendações de adubação e calagem para o Estado de São Paulo. Campinas: Instituto Agronômico \& Fundação IAC. 285p.

RANDHAWA KS; BHAIL AS. 1976. Growth, yield and quality of cauliflower (Brassica oleracea var. botrytis) as influenced by nitrogen, phosphorus and boron. Indian Journal of Horticulture 33: 83-91.

SÁNCHEZ LR; BOTÍA CP; SIRONI JS; SÁNCHEZ AA; CRESPO AP; MARTINEZ CM. 2001. Crecimiento vegetativo y absorción de nutrientes de la coliflor. Investigación Agraria 16: 119-130.

TAKEISHI J; CECÍLIO FILHO AB. 2007. Acúmulo de nutrientes em couve-flor híbrido Verona. In: CONGRESSO BRASILEIRO DE OLERICULTURA, 48. Resumos... Maringá: ABH (CD-ROM).

TRANI PE; PASSOS FA; MELOAMT; TIVELLI SW; BOVI OA; PIMENTEL EC. 2007. Hortaliças e plantas medicinais: manual prático. Boletim Técnico IAC 199: 7-10. 